\title{
Cryo And Cryo-Etch Methods For Quality Preservation Of Hydrogels Imaged At High Magnification By Low Temperature SEM
}

\author{
R.P. Apkarian* and E.R. Wright** \\ *Integrated Microscopy \& Microanalytical Facility / Department of Chemistry, Emory University \\ 1515 Dickey Dr. Atlanta, GA 30322. \\ **Division of Biology, California Institute of Technology Pasedena, CA 91125.
}

Hydrogels are a diverse group of materials that can be engineered from a variety of systems such as proteins, lipid surfactants, and acrylamide-nanoparticles [1,2]. By nature, these materials contain a high water weight percent and have many desirable attributes and applications from photoniccrystals to wound dressings and biomaterials. Hydrogel systems only exhibit their native structure and desired properties when they are in their natural hydration state; a condition often incompatible with high vacuum electron optical systems. Cryo SEM methods are ideally suited for imaging hydrogels because the severe collapse and flattening due to dehydration does not exist in properly frozen specimens. Even environmental SEMs are limited in their ability to assess ultrastructural features of a hydrogel due to the considerable evaporation that occurs during observation in its modest vacuum typically 0.1-10 torr. One group has used slow freezing techniques and cryo-etch LTSEM to purposely form hexagonal ice that leave large pores in alginate hydrogels for bioengineering scaffolds [3]. We rapid freeze hydrogels to attain specimen ice as near vitreous or very small cuboidal ice as possible for the accurate resolution of the macromolecular structure at high magnification with LTSEM. Assuming this can be achieved then the frozen sample surface can be high vacuum etched to sublime away the bulk ice and leave the solid components of the frozen hydrogel such that the surface of the sample would be well preserved and provide "quality" high resolution LTSEM images. For a discussion on freezing and ice see Dubochet [4].

Typically a very small volume of hydrogel $(5-10 \mu 1)$ is as large as one should consider freezing because the larger the volume the slower the freezing, a condition that would result in structural damage due to hexagonal ice formation. We use Balzers gold planchets (BU012 130T) for cryoimmobilization of protein hydrogels in the $1-25 \mathrm{wt} \%$. When performing ethane plunge freezing by hand or using a grid plunging device always do so in a spark arrested hood. Once the specimen has been frozen by plunge or HPF methods, store the samples in $\mathrm{LN}_{2}$ prior to loading onto the cryostage. Be sure to chill all handling tools prior to touching the planchet. The best way to fracture the sample surface is by lightly scraping the planchet surface under $\mathrm{LN}_{2}$ with a prechilled blade. The surface of the sample closest to the cryogen or the HPF planchet sandwich face has the greatest probability of freezing fast enough to attain vitreous ice. Avoid a deep surface fracture as this surface is the best part of your sample. In our in-lens DS-130 FESEM (Topcon) we use a TEM-like cryostage (Gatan CT-3500) equipped with dual protective shutters. Next, we dry nitrogen vent our dedicated high vacuum sputter coater chamber prior to rapid transfer from the $\mathrm{LN}_{2}$ work station. The system is then evacuated to $10^{-7}$ torr to remove all $\mathrm{O}_{2}$. This system can be mimicked by the Alto 2500 cryosystem for near-lens FESEMs. By slowly raising the specimen temperature to between $-105^{\circ} \mathrm{C}$ and $-120^{\circ} \mathrm{C}$ ice can be sublimed out of the hydrogel [2]. The rate of sublimation (etching) is very dependent on vacuum. Balzers provides a guide for $10^{-7}$ torr vacuum systems such that at $-115^{\circ}$ $\mathrm{C}$ the sublimation rate is $\sim 1 \AA / \mathrm{sec}$ and $\sim 100 \AA / \mathrm{sec}$ at $-105^{\circ} \mathrm{C}$. In all hydrogel systems there is the bulk water, the tightly bound water or the hydration shell in contact with the solid phase, and loosely 
bound water that are molecules local to the tightly bound water. Since the hydration properties of each hydrogel vary and differences occur within different regions of the same hydrogel etching times should be experimentally derived. Etching is tricky business because you run the risk of over etching the sample and stripping away the hydration shell, thus freeze drying the hydrogel solid phas leading to artifact. After the etching time is completed, the specimen is returned to $<-180^{\circ} \mathrm{C}$ and sputter coated with 1-2 $\mathrm{nm} \mathrm{Cr}$. The stage is then transferred into the microscope and allowed to equilibrate for $30 \mathrm{~min}$ at the desired imaging temperature. Rapid digital recordings are made at 5-30 $\mathrm{keV}$ in the LTSEM. Attention to preparation details will facilitate quality LTSEM images.

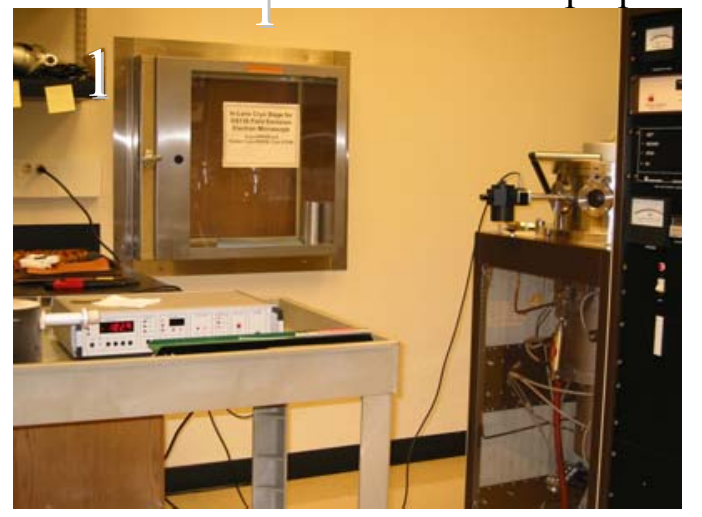

FIG. 1. The Gatan CT-3500 controller monitors the stage temperature during chamber evacuation, etching and $\mathrm{Cr}$ coating.

FIG. 2. Cryoetch LTSEM of 25 wt \% B-9 triblock elastin porous hydrogels. A\&B taken by Cameron Ackerley using a Alto- 2500 cryosystem in a near-lens JEOL 6700 FESEM. C\&D taken with CT-3500 cryorod in a Topcon in-lens FESEM.

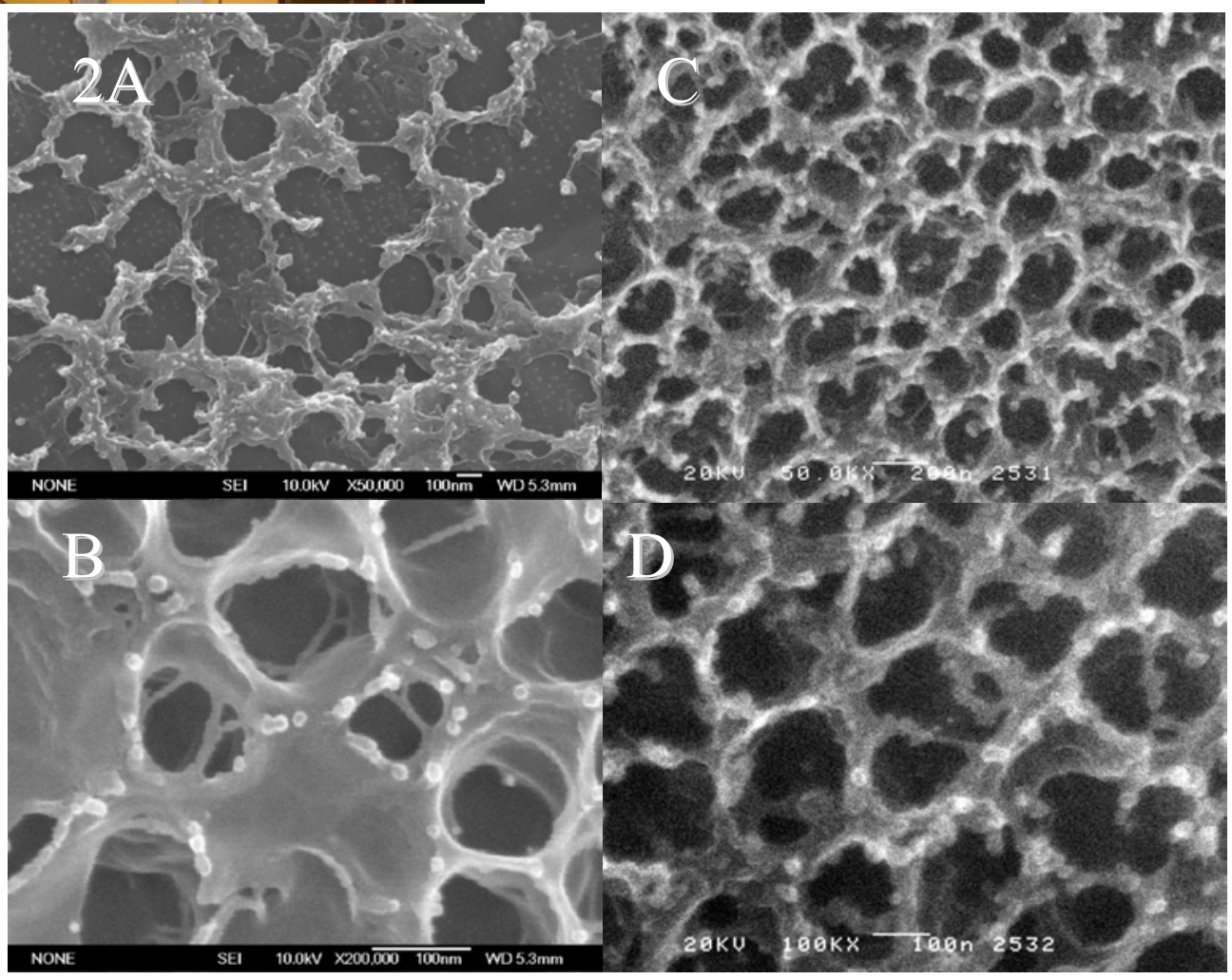

References

1. R.P. Apkarian et al., Microsc. Microanal. 9:4 (2003) 286.

2. E.R. Wright et al., Microsc. Microanal. 9:3 (2003) 171.

3. S. Zmora et al., Biomaterials 23 (2002) 4087.

4. J. Dubochet et al., Quarterly Review of Biophysics 21 (1988) 129. 